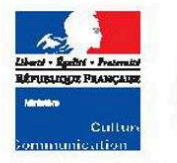

Secrétariat général

Service de la
coordination des

politiques culturelles

et de linnovation

Département

des études,

de la prospective

tes statistiques

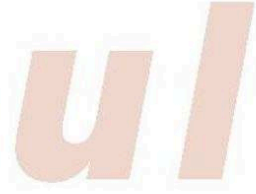

82, rue Saint-Honoré, 75033 Paris cedex 01

중 0140157917 - 图 0140157999

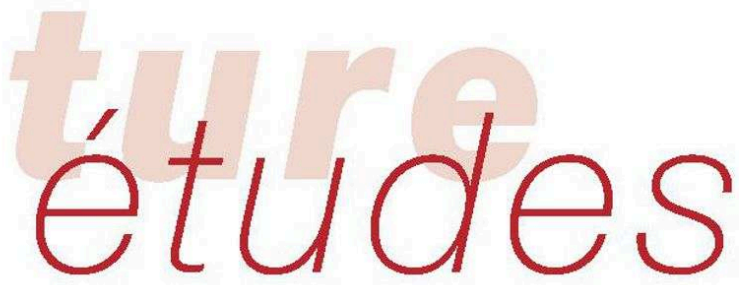

ÉCONOMIE DE LA CULTURE ET DE LA COMMUNICATION

Téléchargeable sur le site hitp://www.culfurecommunication. gouv. if/Etudes-et-statistiques

2013-3

\title{
La presse française en ligne en 2012 : modèles d'affaires et pratiques de financement ${ }^{1}$
}

\author{
Pierre-Jean Benghozi* \\ Inna Lyubareva*
}

\author{
Online press industry in France in 2012 : \\ business models and funding mechanisms
}

L'effet de l'internet sur les industries culturelles a généralement été envisagé, ces dernières années, sous l'angle de la concurrence des formes de consommation dématérialisées et illégales. Pourtant, les transformations à l'œuvre sont plus profondes, comme l'ont montré certains travaux récents menés dans le cadre de l'audiovisuel ou de la musique ${ }^{2}$. Elles tiennen à l'émergence de nouveaux modèles d'affaires, supports de formes de concurrence constamment renouvelées. Dans ces nouvelles configurations, la valeur de l'offre tient souven moins au contenu en soi qu'aux modèles économiques de production et de consommation dans lesquels ils s'inscrivent Multiplier les manières de mettre les contenus culturels à disposition ne constitue pas un simple effet conjoncturel. I] marque des stratégies systématiques d'innovation et d'exploration de modèles d'affaires alternatifs, à même d'assure pérennité et rentabilité : le cas de la presse est tout à fait symptomatique de ce point de vue.

La compréhension de ces phénomènes inédits s'avère d'autant plus importante qu'ils remettent profondément en cause les bases traditionnelles de l'économie de la culture. Dans la plupart des cas, en effet, les industries culturelles reposaient sur des modèles économiques centenaires, établis à leur origine et restés inchangés dans leur principe : vente à l'unité ou location des œuvres et répartition proportionnelle des recettes. C'est notamment le cas du livre, de la presse, de la musique et du cinéma. La prise en compte des nouveaux modèles économiques est déterminante du point de vue de l'action publique. L'importance de la culture en termes d'identité et de rayonnement national tout autant que son poids économique font que les secteurs créatifs ont toujours bénéficié d'une attention particulière des pouvoirs publics. Elle s'est encore accrue ces dernières années avec l'importance grandissante des industries créatives dans l'économie ${ }^{3}$. Les nouveaux modèles d'affaires de la culture portés par l'internet remettent cependant radicalement en cause les formes habituelles d'intervention de l'État, reposant notamment sur le soutien à la création, à la diffusion ou l'aide à la consommation. Le cas de la presse apparait, dans ce contexte, particulièrement intéressant à étudier: il est en effet marqué historiquement par un dispositif complexe d'aides à la presse dont les modalités d'intervention font actuellement l'objet d'un vif débat.

* Pierre-Jean Benghozi (PREG-CRG CNRS - École polytechnique) et Inna Lyubareva (Télécom Bretagne - LuSSI).

1. L'étude est tirée d'un rapport établi pour le Département des études, de la prospective et des statistiques du ministère de la Culture et de la Communication dans le cadre de l'appel à proposition de recherche «Approche par les marchés ou plates-formes multifaces dans le domaine culture et médiatique », les Modèles d'affaires des plates-formes de contenu: étude et typologie à partir du cas de la presse, décembre 2012.

* Avec les contributions de Danièle AtTIAS (Grenwich Consulting), Mehdi FARAJALLAH (PREG-CRG CNRS - École polytechnique), Teaiki Fidéle (PREGCRG CNRS - École polytechnique), Émily HUIBAN (Marsouin), Matthieu LARDEAU (PREG-CRG CNRS École polytechnique), Laure MuseLL) (Télécom ParisTech), Chen ZHI (Université de Singapour).

2. Voir I. LyUbAREVA, P.-J. BenghozI et T. FiDĖLE, "Online Business Models in Creative Industries: Diversity and Structure", Journal of 7nternational Studies in Management and Organization, 2013.

3. Pour une synthèse, voir P. BOvQUILLION et J.-B. LE CORF, les Industries créatives et économie créative dans les rapports officiels européents, Paris, Rapport pour le Département des études, de la prospective et des statistiques du ministère de la Culture et de la Communication, 2010. 


\section{La presse française en ligne en 2012}

Modèles d'affaires et pratiques de financement

Online press industry in France in 2012: business models and funding mechanisms

\section{Pierre-Jean Benghozi et Inna Lyubareva}

Éditeur : Département des études, de la prospective et des statistiques

Lieu d'édition : Paris

Année d'édition : 2013

Date de mise en ligne : 8 juillet 2015

Collection : Culture études

ISBN électronique : 9782111399105

\section{CboOKKS}

http://books.openedition.org

\section{Édition imprimée}

Date de publication : 1 juin 2013

Nombre de pages : 12

\section{Référence électronique}

BENGHOZI, Pierre-Jean ; LYUBAREVA, Inna. La presse française en ligne en 2012 : Modèles d'affaires et pratiques de financement. Nouvelle édition [en ligne]. Paris : Département des études, de la prospective et des statistiques, 2013 (généré le 25 avril 2021). Disponible sur Internet : <http:// books.openedition.org/deps/125>. ISBN : 9782111399105. 

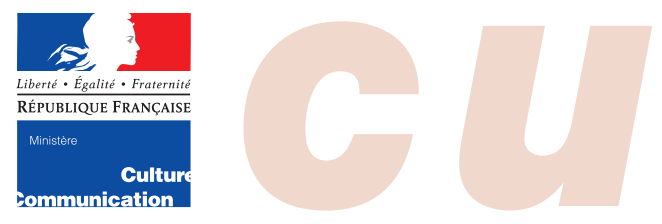

Secrétariat général

Service de la

coordination des

politiques culturelles

et de l'innovation

Département

des études,

de la prospective

et des statistiques

\title{
La presse française en ligne en 2012 : modèles d'affaíres et pratiques de financement ${ }^{1}$
}

\author{
Pierre-Jean Benghozi* \\ Inna Lyubareva*
}

\author{
Online press industry in France in 2012 : \\ business models and funding mechanisms
}

L'effet de l'internet sur les industries culturelles a généralement été envisagé, ces dernières années, sous l'angle de la concurrence des formes de consommation dématérialisées et illégales. Pourtant, les transformations à l'œuvre sont plus profondes, comme l'ont montré certains travaux récents menés dans le cadre de l'audiovisuel ou de la musique ${ }^{2}$. Elles tiennent à l'émergence de nouveaux modèles d'affaires, supports de formes de concurrence constamment renouvelées. Dans ces nouvelles configurations, la valeur de l'offre tient souvent moins au contenu en soi qu' aux modèles économiques de production et de consommation dans lesquels ils s'inscrivent. Multiplier les manières de mettre les contenus culturels à disposition ne constitue pas un simple effet conjoncturel. Il marque des stratégies systématiques d'innovation et d'exploration de modèles d'affaires alternatifs, à même d'assurer pérennité et rentabilité : le cas de la presse est tout à fait symptomatique de ce point de vue.

La compréhension de ces phénomènes inédits s'avère d'autant plus importante qu'ils remettent profondément en cause les bases traditionnelles de l'économie de la culture. Dans la plupart des cas, en effet, les industries culturelles reposaient sur des modèles économiques centenaires, établis à leur origine et restés inchangés dans leur principe : vente à l'unité ou location des œuvres et répartition proportionnelle des recettes. C'est notamment le cas du livre, de la presse, de la musique et du cinéma. La prise en compte des nouveaux modèles économiques est déterminante du point de vue de l'action publique. L'importance de la culture en termes d'identité et de rayonnement national tout autant que son poids économique font que les secteurs créatifs ont toujours bénéficié d'une attention particulière des pouvoirs publics. Elle s'est encore accrue ces dernières années avec l'importance grandissante des industries créatives dans l'économie ${ }^{3}$. Les nouveaux modèles d'affaires de la culture portés par l'internet remettent cependant radicalement en cause les formes habituelles d'intervention de l'État, reposant notamment sur le soutien à la création, à la diffusion ou l'aide à la consommation. Le cas de la presse apparait, dans ce contexte, particulièrement intéressant à étudier : il est en effet marqué historiquement par un dispositif complexe d'aides à la presse dont les modalités d'intervention font actuellement l'objet d'un vif débat.

\footnotetext{
* Pierre-Jean Benghozi (PREG-CRG CNRS - École polytechnique) et Inna Lyubareva (Télécom Bretagne - LUSSI).

1. L'étude est tirée d'un rapport établi pour le Département des études, de la prospective et des statistiques du ministère de la Culture et de la Communication dans le cadre de l'appel à proposition de recherche «Approche par les marchés ou plates-formes multifaces dans le domaine culturel et médiatique », les Modèles d'affaires des plates-formes de contenu : étude et typologie à partir du cas de la presse, décembre 2012.

* Avec les contributions de Danièle AtTiAs (Grenwich Consulting), Mehdi FARAJALlah (PREG-CRG CNRS - École polytechnique), Teaiki FidèLE (PREGCRG CNRS - École polytechnique), Émily HUIBAN (Marsouin), Matthieu LARDEAU (PREG-CRG CNRS École polytechnique), Laure Muselli (Télécom ParisTech), Chen ZHI (Université de Singapour).

2. Voir I. Lyubareva, P.-J. Benghozi et T. Fidèle, “Online Business Models in Creative Industries: Diversity and Structure”, Journal of International Studies in Management and Organization, 2013.

3. Pour une synthèse, voir P. BOUQUILLION et J.-B. LE CoRF, les Industries créatives et économie créative dans les rapports officiels européens, Paris, Rapport pour le Département des études, de la prospective et des statistiques du ministère de la Culture et de la Communication, 2010.
} 


\section{Contexte}

On observe aujourd'hui, dans le secteur de la presse écrite, une redistribution des supports, papier et numérique, qui, bien que moins violente que dans la musique, n'en est pas moins incontestable. Ainsi, la presse française représentait, en 2012, 3963000 exemplaires (en baisse annuelle de 3,8\%) vendus par 845 titres et 7237000000 visites internet (en hausse annuelle de $21 \%$ ) sur 127 sites de presse 4 .

Cette évolution s'accompagne d'une complexification du paysage de la presse, marquée par la variété des formats et par des formes de concurrence inédites entre titres traditionnels et plates-formes d'information en ligne. Face à une situation qui ne semble pas stabilisée, analystes et praticiens s'interrogent sur les principes guidant cette nouvelle économie de la presse.

La littérature académique en management stratégique s'est penchée, depuis quelques années, sur le rôle central des modèles d'affaires dans les évolutions associées à l'économie numérique ${ }^{5}$. Dématérialisation des supports, forfaitisation des achats, gratuité sont les caractéristiques communes de cette nouvelle économie. Elles expliquent l'arrivée actuelle des offreurs de technologie ou d'opérateurs de télécommunication sur le marché des contenus et le bouleversement structurel des modèles d'affaires et de l'architecture des filières économiques de la culture. La presse ne fait pas exception : elle cumule à la fois une baisse significative de ses revenus et une montée en puissance tout aussi nette de ses investissements dans les technologies de l'information et de la communication (TIC) ${ }^{6}$.

Les nouvelles configurations appellent, de la part des acteurs des médias, des stratégies inédites et de nouvelles manières de construire leurs positions compétitives. La concurrence entre titres s'opère moins sur le projet éditorial que sur les modèles d'affaires et sur les modalités de mise à disposition de l'information. Un foisonnement de contenus dont les modalités variées de mise à disposition remettent en jeu les dispositifs traditionnels de rémunération des journalistes, éditeurs et diffuseurs et déstabilise profondément le secteur de la presse. Sur les titres de journaux en ligne par exemple, articles de journalistes professionnels, dépêches d'agence et textes de blog sont proposés sur un même niveau. L'effet est d'autant plus puissant que l'offre en ligne se double aussi, pour des raisons différentes, de l'émergence de propositions gratuites radicalement nouvelles dans les circuits traditionnels.

Il s'agit donc de spécifier les modèles d'affaires dominants des sites de presse en ligne, et de comprendre les trajectoires d'évolution associées dans l'économie de la presse, notamment dans le cas de la gratuité. L'économie de la presse est décrite à partir des caractéristiques observables des principaux modèles d'organisation sur la base desquelles est construite une typologie des modèles d'affaires dominants des producteurs de contenu de la presse.

\section{Premiers Éléments DE DIFFÉRENCIATION DES MODÈLES DE LA PRESSE EN LIGNE}

L'un des principaux effets de la numérisation est la possibilité de démultiplier les modalités de mise à disposition et de commercialisation des contenus grâce aux possibilités ouvertes par la dématérialisation et la délinéarisation de ces contenus ${ }^{7}$. L'étude de l'offre en ligne proposée par 149 titres français montre ainsi, d'emblée, la grande disparité en termes de contenu, de services offerts, de stratégies de tarification, de dispositifs d'interactions avec les lecteurs et de modalités de distribution.

\section{Structure de l'offre}

Les structures d'offre existantes s'appuient à la fois sur les nouvelles possibilités de contribution ouvertes aux lecteurs et sur la customisation des interfaces et des contenus. Les titres de presse tendent ainsi à compléter en ligne leur offre d'articles par des contenus extérieurs issus de bloggeurs invités (blogs rédactionnels) et de lecteurs (commentaires et blogs). La contribution des lecteurs à la production de contenu ne se réduit pas aux contenus textuels : création et mise en ligne d'images, de sons et de vidéos font, dans certains cas, partie intégrante de l'offre (on parle alors de contenus générés par les contributeurs ou Users Generated Contents). Ce faisant, les titres n'offrent plus un support indivisible - le journal papier - agrégeant de manière unique les contenus produits. Ils s'ouvrent désormais à de nouveaux services comme la navigation personnalisée ${ }^{8}$ et les flux RSS thématiques permettant de proposer aux lecteurs le contenu adapté individuellement à leurs besoins et à leurs goûts. Les offres en ligne enrichissent également les contenus en informant leurs lecteurs des choix et intérêts d'autres lecteurs grâce aux dispositifs statistiques (classement des articles les plus lus, les plus commentés, les plus partagés...). Enfin, la fréquence continue de publication constitue une innovation importante rendue possible qui modifie profondément les pra-

4. Source : OJD, Association pour le contrôle et la diffusion des médias, 2013, http://observatoire.ojd.com/

5. B. Mahadevan, "Business Models for Internet-Based E-Commerce: An Anatomy", California Management Review, 2000, vol. 42, no 4, p. 55-69 ;

P.-J. BeNGHOZI, « Relations interentreprises et nouveaux modèles d'affaires », Revue économique, Numéro spécial «Économie de 1'Internet », 2001, 52, p. 167-190; S. M. Shafer, H. J. Smith, J. C. Linder, The Power of Business Models, Business Horizons, 2005, vol. 48, Issue 3, p. 199-207; H. Chesbrough, "Why Companies Should Have Open Business Models", MIT Sloan Management Review, 2007, 48 (2), p. 22-28 ; A. OsterwALDER, Y. Pigneur, Business Model Generation: A Handbook for Visionaries, Game Changers, and Challengers, John Wiley\&Sons, 2010 ; B. DemIL et X. LECOCQ, "Business Model Evolution: In Search of Dynamic Consistency", Long Range Planning, 2010, vol. 43, no 2-3, avril-juin, p. $227-246$; C. ZotT, R. Аміт, "The Fit Between Product Market Strategy and Business Model: Implications for Firm Performance", Strategic Management Journal, 2008, 29 (1), p. 1-26 ; D. J. TeECE, Dynamic Capabilities and Strategic Management: Organizing for Innovation and Growth, New York, Oxford University Press, 2009.

6. http://www.enpa.be/uploads/Martin/enpa_media.pdf

7. E. Dagiral, S. Parasie, «Presse en ligne: où en est la recherche ?», Réseaux, 2010, ${ }^{\circ}$ 160-161, Numéro spécial «Presse en ligne», p. 13-42 ; D. AtTias, l'Impact d'internet sur l'économie de la presse : quel chemin vers la profitabilité ?, thèse de sciences économiques, Université de Paris Ouest/Nanterre La Défense, 2007.

8. La navigation personnalisée est la possibilité pour l'utilisateur de gérer les thématiques qu'il a envie de voir apparaître sur la page d'accueil du site. 
tiques des lecteurs amenés à multiplier les contacts avec le site d'un titre plutôt que de se contenter d'un acte d'achat occasionnel, quotidien dans le meilleur des cas.

\section{Monétisation}

La variété des formes de monétisation des contenus est une autre caractéristique. Outre la stratégie de tarification par abonnement, largement répandue dans la presse, une grande diversité d'options de tarification s'est développée avec les technologies de l'information et de la communication. Ainsi, les articles et les services associés peuvent être soit vendus à l'unité, soit offerts gratuitement pendant une période limitée (crédit temps : numéro accessible en ligne pendant 24 heures) ou en quantité limitée (crédit quantité : accès gratuit pour trois articles). Les éditeurs utilisent également le service de consommation offline (articles en PDF) et l'accès aux archives numériques comme sources supplémentaires de revenus.

\section{Interactivité}

L'évolution des interfaces proposées aux lecteurs et les nouvelles structurations du lectorat constituées autour de l'interactivité sont une autre caractéristique de la presse en ligne. Les nouvelles technologies permettent l'accès à une large gamme des dispositifs d'interaction avec les lecteurs : dispositifs d'évaluation des articles («j'aime»), utilisés par les éditeurs de contenus pour mieux cibler leur production ; constitution d'un réseau social propre au titre de presse et réservé uniquement à ses lecteurs ${ }^{9}$. Dans la même lignée, la création d'une page sur un des réseaux sociaux existants (par exemple Facebook) représente une nouvelle option dans l'interaction entre l'éditeur et ses consommateurs. L'ensemble de ces dispositifs d'interaction est souvent complété par les forums de discussion.

\section{Diffusion}

Enfin, les nouvelles technologies de l'information ont créé de nouveaux canaux de distribution auparavant inaccessibles aux acteurs de l'industrie de la presse : les réseaux sociaux (par exemple, Facebook via la fonctionnalité de partage des articles), les kiosques numériques où le titre de presse est généralement disponible en abonnement ou à l'unité sur plusieurs supports (ordinateurs, tablettes, smartphones, papier).

\section{TrOIS ClASSES DE MODÈLES D'AFFAIRES IDENTIFIABLES}

Des formes dominantes se profilent-elles derrière ce large éventail d'opportunités nouvelles, et, le cas échéant, quelles sont leurs lignes de force? L'étude économétrique menée en construisant et en exploitant une base de données inédite de 149 titres de presse en ligne permet effectivement d'identifier trois classes distinctes de modèles d'affaires de la presse en ligne (graphique 1).

\section{Graphique 1 - Trois modèles d'affaires de titres de presse en ligne}

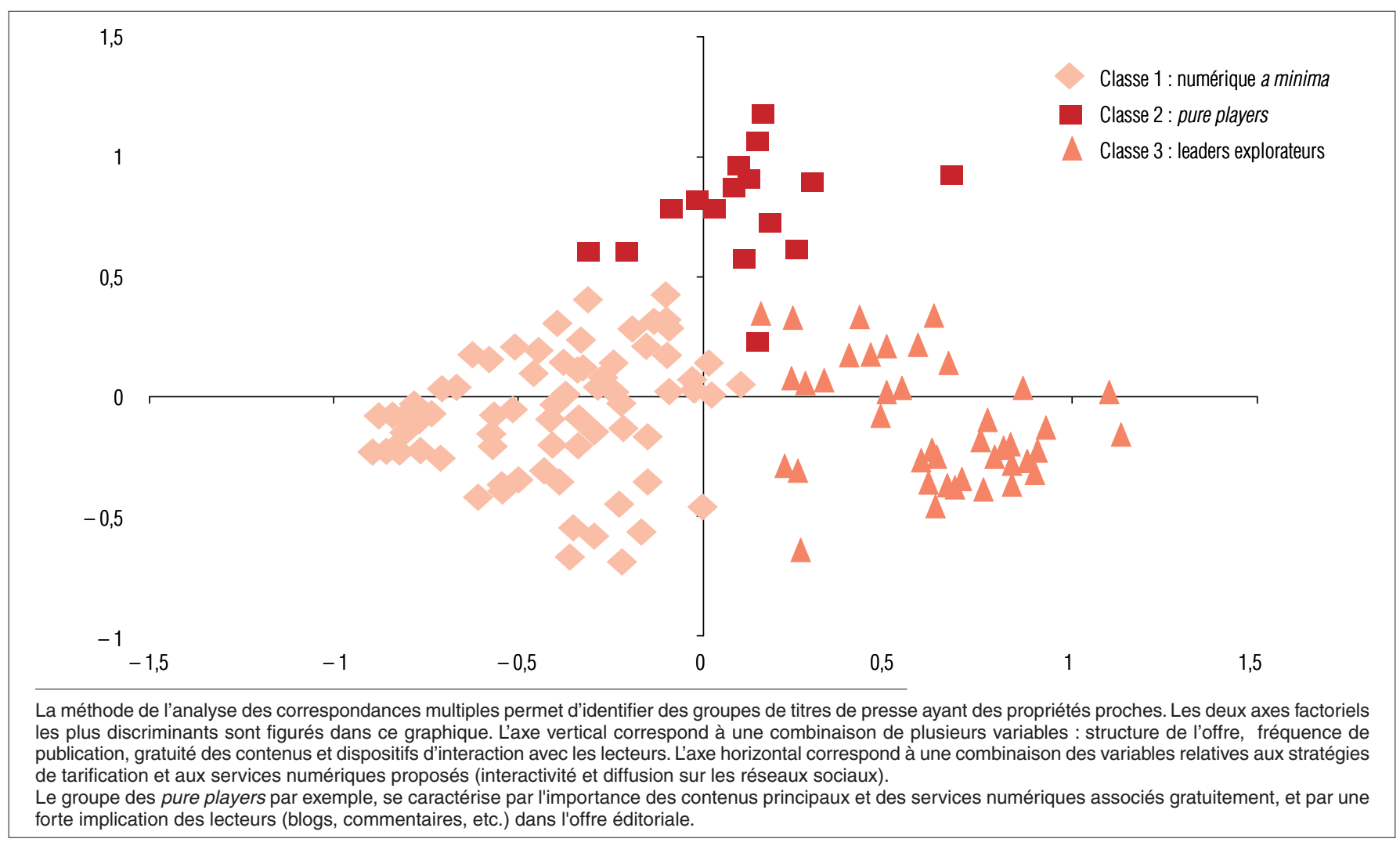

Source : DEPS, Ministère de la Culture et de la Communication, 2013

9. Les lecteurs peuvent fédérer un groupe d'individus autour d'un thème ou d'un travail commun comme dans le cas de www.lesinrocks.com 
Tableau 1 -Caractéristiques de trois classes de modèles d'affaires (dans l'ordre de leur importance pour la classe respective)

\section{1 - Numérique a minima \\ - Absence de statistiques sur les articles (lus/commentés/partagés) \\ - Absence de compte Twitter \\ - Absence de partage de contenu sur les réseaux sociaux \\ - Absence d'évaluation des articles \\ - Absence de flux RSS \\ - Absence de liens sponsorisés comme modèle de revenus}

\section{2 - Pure players}

- Fréquence de publication en continu

- Le site n'est pas issu d'un acteur traditionnel des médias

- Accès aux archives gratuit

- Statistiques sur les articles (les plus lus, commentés, etc.)

- Existence d'autres services payants à l'unité (services indépendants des contenus principaux)

- Pas de vente en ligne : le contenu en ligne et les services associés sont gratuits

\section{3 - Leaders explorateurs}

- Tarification par abonnement web et web+

- Tarification par crédit temps/crédit quantité

- Tarification par vente à l'unité

- Consommation offline payante

- Accès payant aux archives

- Compte Twitter

Source : DEPS, Ministère de la Culture et de la Communication, 2013.

Ces trois classes se distinguent plus particulièrement par des caractéristiques distinctives ${ }^{10}$ au regard des variables identifiées (tableau 1). Elles sont désignées comme suit: «numérique a minima» pour la première, «pure players » pour la deuxième et « leaders explorateurs » pour la troisième.

\section{Numérique a minima}

La classe dite «numérique $a$ minima » regroupe des titres de presse pour lesquels l'ouverture au numérique est relativement réduite ou dont les opportunités restent faiblement exploitées. Contrairement à certains titres qui s'efforcent de tirer tout le potentiel possible de l'internet pour construire leur audience, structurer une communauté de lecteurs en se positionnant comme un portail de référence dans leur domaine, les titres présents dans cette classe se limitent à une utilisation minimale de l'internet, se contentant parfois de sites très pauvres qui peuvent être de simples vitrines. L'interaction avec les lecteurs demeure faible. On observe, par exemple, qu'au total un quart $(24 \%)$ des sites de presse en ligne disposent seulement d'une page vitrine Facebook ${ }^{11}$, et qu'un peu plus des trois quarts (78\%) de ceux-ci appartiennent à la classe «numérique $a$ minima ». Le système de commentaires des articles est peu présent dans l'offre de cette classe : seulement $28 \%$ des 149 sites de presse étudiés n'emploient pas ce dispositif, mais la plupart $(95 \%)$ appartiennent à cette première classe ${ }^{12}$.

Une autre illustration de ce faible engagement dans le numérique apparaît dans le traitement des archives en ligne : $59 \%$ des sites de cette classe ne donnent pas accès aux archives, même moyennant paiement, alors que ce n'est le cas que pour une infime minorité des titres des autres classes $(6 \%$ pour la classe des pure players et $10 \%$ pour la troisième classe des leaders explorateurs).

Cette première classe fait très faiblement appel aux liens sponsorisés comme modèle de revenu : parmi les 149 titres analysés, $39 \%$ n'ont pas de liens sponsorisés dont $88 \%$ appartiennent à la classe numérique a minima.

Ainsi, cette classe de modèles d'affaires est d'abord mue par la volonté de protéger un modèle économique traditionnel, rentable et adapté, dont les titres de presse redoutent la mise en péril et la cannibalisation par le numérique. Ces titres de presse ne se lancent sur l'internet que par suivisme et par obligation, et se montrent peu concernés par l'innovation technologique. Ils s'efforcent avant tout de conserver leur modèle historique, sans chercher à développer les services et fonctionnalités associées au nouveau média numérique.

Parmi les titres appartenant à cette classe de modèles d'affaires on peut citer: Gala, Télé 2 semaines, La Presse de la Manche ou Métro, et plus particulièrement ceux qui en sont les plus représentatifs : Top vélo, Lutte ouvrière, Le Tarn libre, Le Courrier du pays de Retz ainsi que les titres comme Bretons ou Le Canard enchaîné (voir tableau 6).

Les titres de presse appartenant à cette première classe numérique a minima ont en commun la fréquence de parution : pour près des trois quarts $(72 \%)$, ce sont des titres de presse papier (bi)hebdomadaire, (bi)mensuelle et trimestrielle. Sur l'ensemble des trois classes réunies, on observe d'ailleurs que plus de la moitié (52\%) des titres de presse ayant une fréquence de parution autre que quotidienne ou en continu (pour le web) appartiennent majoritairement $(75 \%)$ à la première classe. En outre, la proportion de magazines est plus importante dans la classe numérique a minima que dans les deux autres classes : $71 \%$ des magazines dans l'échantillon global appartiennent à la première classe des modèles d'affaires (les autres $29 \%$ appartenant à la troisième classe).

10. Le tableau 1 présente uniquement les caractéristiques les plus significatives de chaque classe. Ces caractéristiques sont révélées par la comparaison entre ces trois classes. Par exemple, «pas de liens sponsorisés comme modèle de revenu » dans la classe 1 signifie que, dans cette classe, le nombre d'acteurs de la presse utilisant ce modèle de revenu est particulièrement faible par rapport à deux autres classes et à la distribution de cette variable dans l'échantillon. Il est donc important de noter que la liste des caractéristiques de chacune des classes n'est pas exhaustive.

11. Les pages vitrines ne sont pas très élaborées, elles reprennent souvent le texte de la page Wikipedia correspondant au site de presse.

12. En termes de la structure de l'offre et des dispositifs d'interaction, le titre Les Inrockuptibles de la classe 1 est l'un des rares cas de magazine publiant en ligne en continu et enrichissant cette offre par des dispositifs d'évaluation des articles, commentaires des lecteurs, blogs rédactionnels et interaction via les réseaux sociaux. 


\section{Pure players}

La deuxième classe de modèle de presse en ligne, qualifiée de «pure players», regroupe les nouveaux acteurs de la presse, ceux qui se donnent comme objectif de construire des modèles économiques originaux et cohérents, à même de leur permettre d'établir une position inédite et stable. Ainsi, l'intégralité des sites d'acteurs en ligne présents dans l'échantillon appartient à cette classe des pure players; à l'inverse, seuls deux des sites de journaux de la presse papier traditionnelle (20 Minutes et France Soir ${ }^{13}$ ) partagent les caractéristiques de cette classe. Parmi les titres les plus représentatifs, on peut citer, entre autres, France Net Info, Atlantico, Slate, Quoi.info et Rue89.

La classe des pure players se distingue par la richesse des contenus principaux et des services associés offerts gratuitement, et par une forte implication des lecteurs dans la construction de l'offre éditoriale. Cette dimension de gratuité ne tient pas ici uniquement à l'usage des liens sponsorisés mais elle s'articule également avec la commercialisation de produits et services indépendants des contenus principaux (articles de presse): bandes dessinées, soutien scolaire, dictionnaires, etc. Dans le même temps, certains titres, à l'image de Médiapart, emblématiques de ces nouveaux acteurs de la presse ont réussi à construire des modèles économiques originaux par la variété des mécanismes de tarification directe des contenus : crédit temps/quantité et différents abonnements en ligne. Ils restent, pour autant, dans le même cadre éditorial de production d'informations. Ils reposent sur une vision commune de la presse mais innovent essentiellement sur le modèle économique, le mode de production des informations journalistiques et la construction d'une nouvelle relation avec un réseau de lecteurs et de contributeurs.

\section{Leaders explorateurs}

La troisième classe des «leaders explorateurs » est représentée par des grands quotidiens - tels que L'Équipe, La Tribune, L'Humanité, Libération, Le Figaro et Le Journal du dimanche - qui ont le souci de protéger leur position de leader et sont conscients de devoir, pour cela, explorer les nouvelles configurations ouvertes sur l'internet. Cette classe est composée de $80 \%$ de journaux et de $63 \%$ de presse quotidienne : elle regroupe notamment les titres les plus diffusés par exemplaire (tableau 2).

\section{Tableau 2 - Les dix titres de presse les plus diffusés (nombre d'exemplaires vendus) en 2012}

\begin{tabular}{|c|c|}
\hline \multicolumn{2}{|l|}{ Diffusion France payée par numéro } \\
\hline Ouest France & 749200 \\
\hline Le Figaro & 323300 \\
\hline Le Monde & 288100 \\
\hline Sud Ouest & 280460 \\
\hline Le Parisien & 274900 \\
\hline L'Équipe Édition générale & 274800 \\
\hline La Voix du Nord & 251600 \\
\hline Le Dauphiné libéré & 223800 \\
\hline Le Télégramme & 203300 \\
\hline Le Progrès - La Tribune/Le Progrès & 198300 \\
\hline
\end{tabular}

Pour ces raisons, les titres de presse des leaders explorateurs restent très fortement dépendants des modes traditionnels de rémunération : ces journaux s'efforcent de générer de nouveaux revenus, tout en préservant les anciens (ou en évitant d'accélérer la chute des revenus papier).

Par rapport au modèle traditionnel, l'originalité de la démarche en ligne se traduit tout d'abord dans les nouvelles formes de tarification des contenus principaux et la commercialisation des services associés: dispositifs de crédittemps/quantité, abonnement en ligne complété par les offres mobile et tablette (web+), vente des archives numériques et du service de consommation offline.

Ensuite, le financement par les liens sponsorisés, quoique très présent aussi parmi les pure players $(65 \%$ des titres des pure players emploient ce mode de financement), est la stratégie la plus répandue dans la troisième classe : $98 \%$ des leaders explorateurs y ont recours. Il est sans doute d'autant plus présent pour ces titres qu'il constitue un prolongement naturel des modes traditionnels de financement de la presse par la publicité, à la fois dans le modèle d'affaires et, le cas échéant, sans doute dans l'organisation des régies publicitaires de la presse.

Cette troisième classe de modèle d'affaires se caractérise aussi par une forte interaction avec les lecteurs par l'intermédiaire des réseaux sociaux, des systèmes des commentaires et des statistiques des articles. Néanmoins, l'implication des lecteurs ne concerne pas forcément le processus de production des contenus principaux, qui reste souvent très centralisé. Ainsi, peu de ces titres disposent des blogs de lecteurs ou d'autres formes de contenus générés par les utilisateurs (UGC). C'est même là l'une des différences notables avec les pure players. Enfin, une des propriétés importantes des leaders explorateurs concerne l'orientation forte vers la personnalisation de l'offre à travers les flux RSS et le service de navigation personnalisée (tableaux 3 et 4). $60 \%$ des acteurs de cette classe offrent des services de personnalisation gratuitement.

\section{Tableau 3 - Personnalisation du contenu : flux RSS}

\begin{tabular}{|l|c|c|c|c|c|}
\hline $\begin{array}{l}\text { Nombre de flux } \\
\text { Rss individualisés }\end{array}$ & 0 & $1-11$ & $12-26$ & $\mathbf{2 7 - 4 5}$ & $\begin{array}{c}\text { Plus de } \\
\mathbf{4 5}\end{array}$ \\
$\begin{array}{l}\text { Classe 1 } \\
\quad \text { Numérique a minima }\end{array}$ & 85 & 39 & 30 & 9 & 0 \\
$\begin{array}{l}\text { Classe 2 } \\
\quad \text { Pure players }\end{array}$ \\
$\begin{array}{l}\text { Classe 3 } \\
\text { Leaders explorateurs }\end{array}$ & 4 & 21 & 15 & 18 & 0 \\
\hline
\end{tabular}

Source : DEPS, Ministère de la Culture et de la Communication, 2013.

Tableau 4-Personnalisation du contenu : navigation

\begin{tabular}{|c|c|c|}
\hline Classes & $\begin{array}{l}\text { Absence de navigation } \\
\text { personnalisée }\end{array}$ & $\begin{array}{l}\text { Navigation } \\
\text { personnalisée }\end{array}$ \\
\hline Numérique a minima & 66 & 33 \\
\hline Pure players & 16 & 2 \\
\hline Leaders explorateurs & 17 & 66 \\
\hline
\end{tabular}

Source : DEPS, Ministère de la Culture et de la Communication, 2013.

13. Il est de ce point de vue particulièrement significatif de noter que France Soir est justement devenu totalement en ligne en 2012. 


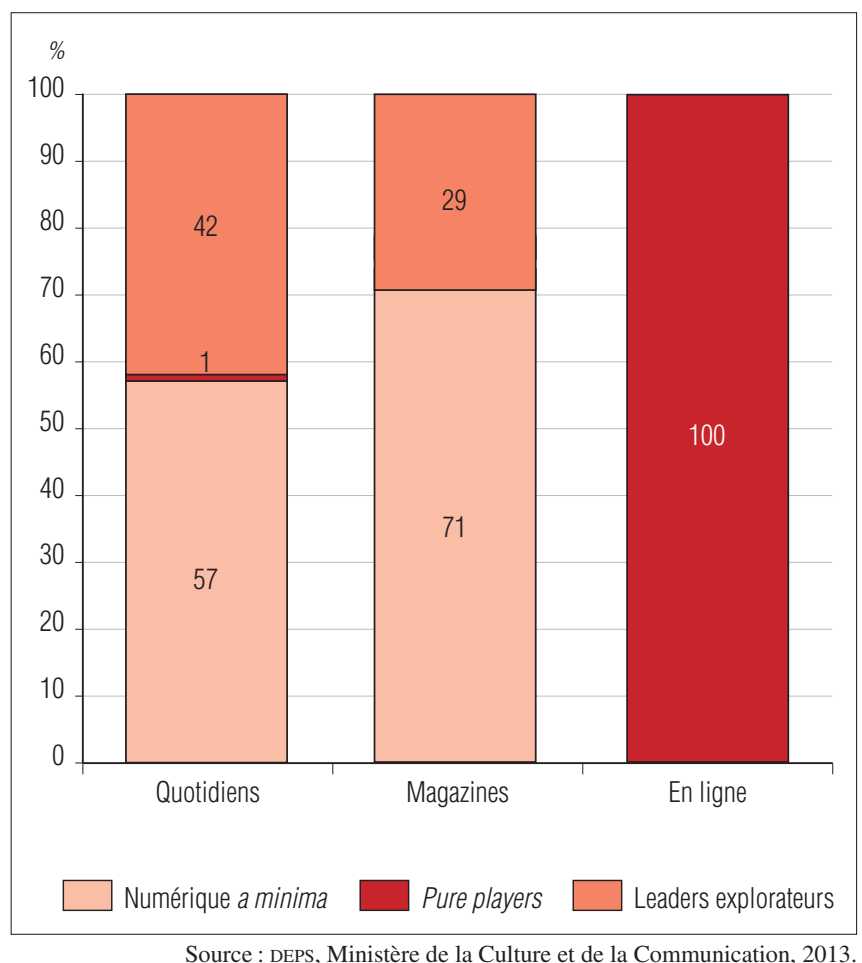

La multiplication des modèles ou des manières de mettre les contenus de la presse à disposition traduit des stratégies d'exploration de modèles d'affaires alternatifs capables d'assurer pérennité et rentabilité dans un environnement nouveau. Cela explique les innombrables démarches d'essais et d'erreurs ou d'aller-retour qu'opèrent les fournisseurs de contenus en ligne pour tester des solutions et trouver ce qu'ils espèrent être le modèle ad hoc. Les cas du Monde ou du Parisien sont tout à fait symptomatiques de ce point de vue : la situation de ces journaux manifeste les conséquences que peut avoir un tel choix sur l'organisation interne du journal et la structuration de sa rédaction contrainte de concilier, autour du multimédia, des objectifs et des modes de production parfois complètement opposés. La classe des leaders explorateurs caractérise bien ces acteurs de médias traditionnels s'efforçant de faire évoluer leur offre en maintenant une grande richesse d'éditorialisation et de contenus : des grands titres qui se lancent résolument sur l'internet pour protéger leur position de leader, en n'hésitant pas à explorer différents types d'offres et configurations.

\section{COMPARAISON ET SPÉCIFICITÉ DES DIFFÉRENTS MODÈLES D’AFFAIRES}

Le poids de la presse imprimée sur les choix numériques stratégiques se retrouve, de façon plus générale, dans l'empreinte de son organisation sur les différentes classes de modèles d'affaires en ligne.

Un premier constat est frappant. La catégorie du titre (quotidien, magazine ou nouvel entrant), l'appartenance de l'acteur au média papier et la périodicité du titre papier (le cas échéant) constituent des variables largement discriminantes pour les dans les trois classes

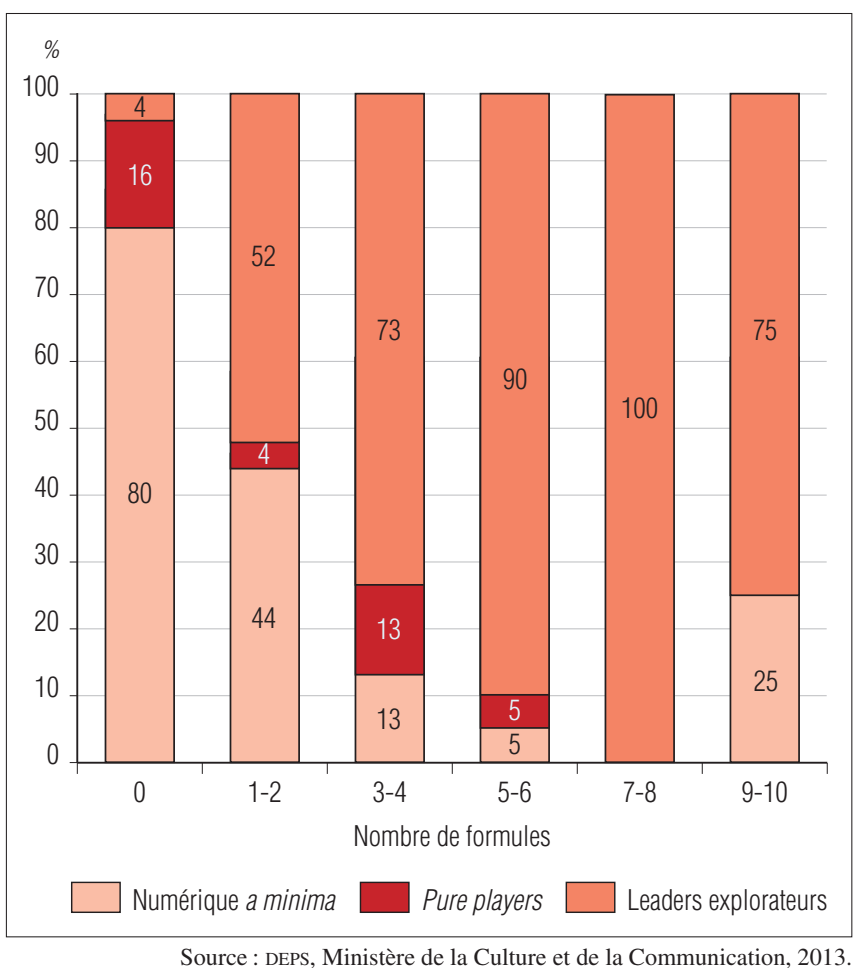

trois classes, alors même que ces variables perdent justement tout leur sens en ligne (graphique 2).

À l'inverse, certaines caractéristiques comme l'aire géographique de diffusion ou d'origine des informations ne jouent pas de rôle structurant important. L'échantillon comprend ainsi $55 \%$ de titres de presse nationale et $45 \%$ de titres régionaux, qui sont répartis de façon homogène entre les numériques a minima (52\% des titres nationaux et $48 \%$ des titres régionaux) et les leaders explorateurs $(45 \%$ et $55 \%$ respectivement), alors que l'on aurait pu imaginer que la structuration des titres par genre (sport, communautés locales, géographiques ou culturelles) favorisait au contraire des modalités d'échanges en ligne déjà présentes dans de nombreuses communautés d'intérêt.

La variété des formes de tarification en ligne et des formules d'abonnement constitue une autre variable particulièrement marquante selon les classes (graphique 3). Ce sont principalement les leaders explorateurs qui offrent à leurs clients une large gamme de formules en ligne et cette stratégie de diversification de l'offre numérique n'est pas une simple répli-

Tableau 5 - Prix moyens des titres de presse, 2012

En euros

\begin{tabular}{|lcc|} 
& & \\
& $\begin{array}{c}\text { Numérique } \\
\text { a minima }\end{array}$ & $\begin{array}{c}\text { Leaders } \\
\text { explorateurs }\end{array}$ \\
Prix d'un abonnement papier min. & 77 & 265 \\
Prix d'un abonnement papier max. & 102 & 311 \\
Prix à l'unité web & 0,75 & 1,5 \\
Prix abonnement web/web+ min. & 78 & 145 \\
Prix abonnement web/web+ max. & 106 & 255 \\
& & \\
\hline
\end{tabular}

Source : DEPS, Ministère de la Culture et de la Communication, 2013. 
cation de la tarification de la version papier. Ainsi par exemple, alors que près de deux tiers $(64 \%)$ des titres de presse de la classe numérique a minima proposent des abonnements diversifiés pour la version papier, les titres de cette classe n'offrent qu'un nombre très restreint de formules en ligne au lecteur. Enfin, on remarque que les titres de presse des leaders explorateurs affichent des prix moyens, web et papier, bien plus élevés que ceux des autres classes (tableau 5).

\section{E-GRATUITÉ ET E-RENTABILITÉ : OPPORTUNITÉS ET SOURCES D'INNOVATION?}

L'omniprésence de l'offre d'informations gratuites sur les sites de presse, quelle que soit leur classe, traduit l'importance renouvelée d'une économie de la gratuité qui se nourrit des dimensions spécifiques du numérique : importance des contenus offerts, variété des informations, démultiplication des modalités d'accès et de tarification. À l'exception de quelques cas $^{14}$ qui représentent plutôt une exception aux règles générales, la numérisation des titres de presse s'accompagne souvent de la gratuité, pour une partie au moins de leur contenu.

Mais si la question de la gratuité dans l'industrie de la presse écrite est devenue centrale dans le débat actuel, c'est que plusieurs modèles économiques très différents se sont mis en place reposant chacun sur des formes variées de gratuité pour concilier e-gratuité et e-rentabilité.

La presse ne déroge pas, de ce point de vue, aux constats dressés dans d'autres domaines d'une économie numérique. L'articulation y est constante entre dimensions marchandes et non marchandes dans la manière de structurer l'offre et la dif-

\section{Graphique 4-Différents modèles de gratuité identifiés auprès de 149 titres de presse}

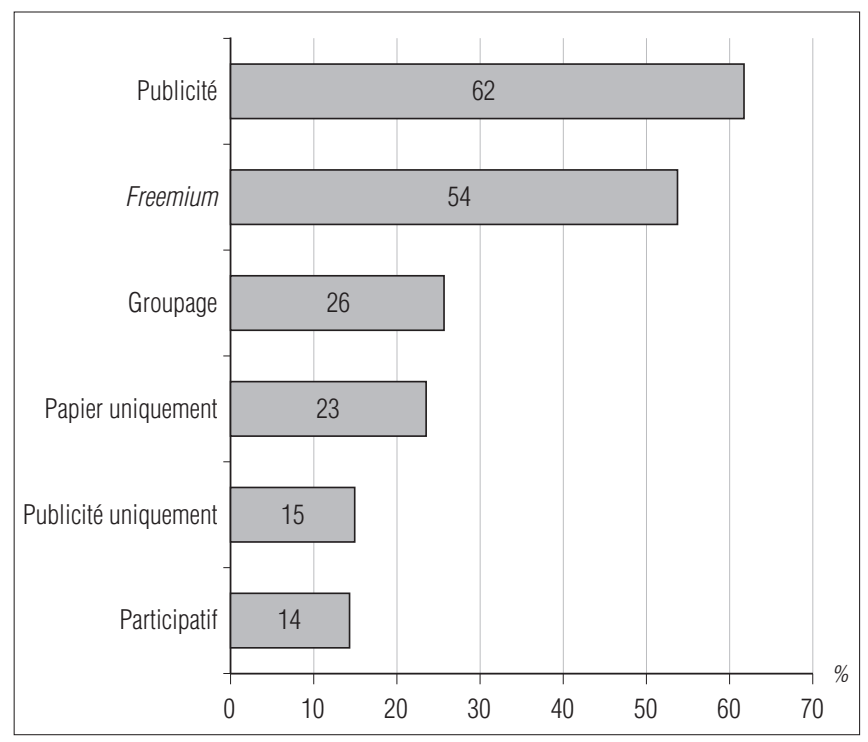

Source : DEPS, Ministère de la Culture et de la Communication, 2013. fusion de contenus comme dans celle d'organiser l'implication des différents contributeurs. Les enjeux cristallisés par la question de la gratuité sont liés à l'histoire de la naissance et du développement du réseau internet, ancré depuis ses débuts dans une culture d'échange et de coopération. L'articulation entre gratuité et valorisation économique est le moteur du web car toutes les prestations gratuites reposent sur des financements masqués: infrastructures subventionnées par la puissance publique, capitalisations non rémunérées, non-paiement des contenus, contribution volontaire et bénévole des clients... tous peuvent être qualifiés de gratuits, mais gratuits pour les vendeurs ou pour les acheteurs?

Le secteur culturel est profondément déstabilisé par ce foisonnement de contenus gratuits car les modalités de ces offres remettent en jeu les modes traditionnels de rémunération des producteurs, des diffuseurs et des auteurs. L'effet est d'autant plus puissant que cette offre en ligne se double aussi, pour diverses raisons, de l'émergence de certaines propositions gratuites radicalement nouvelles dans les circuits traditionnels. L'émergence de la presse papier gratuite est, de ce point de vue, le plus emblématique. Elle existait depuis très longtemps dans la presse de petites annonces, mais sous des formes à très faible contenu éditorial et relevant de marchés de niches ne concurrençant pas directement la presse payante. La vraie rupture se produit en 2002 en France lorsque le journal Métro est lancé, rapidement suivi par 20 Minutes, sur un registre voisin et concurrent des quotidiens d'information générale.

L'étude permet d'identifier, parmi les nombreuses modalités de gratuité des biens culturels répertoriés dans la littérature $^{15}$, les grands modèles dominants dans la presse française. Plus précisément, quatre modèles ont été identifiés : publicité, groupage, freemium et modèle participatif (graphique 4). Deux constats s'imposent d'emblée. Tout d'abord, la publicité en ligne constitue la forme la plus répandue parmi les quatre modèles (62\% des titres y ont recours), mais elle est rarement employée en tant que source unique des revenus : seulement $15 \%$ des titres de presse analysés se fondent uniquement sur les ressources publicitaires. Ensuite, l'étude montre qu'un nombre non négligeable d'acteurs de la presse (23\%) finance la gratuité des éditions en ligne uniquement par la version papier de leurs titres, sans recours aux modalités de financement en ligne.

\section{La publicité}

La publicité est l'une des modalités les plus connues et la plus fréquemment mobilisée dans les médias, notamment à la radio et la télévision. Son principe repose sur la valorisation d'une audience auprès d'annonceurs tiers. Des contenus fournis gratuitement attirent des spectateurs ou auditeurs qui sont autant de consommateurs potentiels, aux caractéristiques plus ou moins identifiables selon les programmes fournis. Le titre peut alors commercialiser des espaces publicitaires auprès de fournisseurs de biens et services soucieux de promouvoir leurs produits sur la cible ainsi construite. Le poids de ce modèle se manifeste largement dans la presse en ligne où l'on identifie

14. Pour quelques exceptions comme Médiapart, Minute, Présent ou Rivarol, les contenus sont strictement réservés aux abonnés.

15. Voir notamment C. Anderson, The Long Tail. Why the Future of Business is Selling Less of More, New York, Hyperion, 2006 ; C. SHAPIRO et H. R. VARIAN, Économie de l'information, Paris, De Boeck Université, 1999. 
l'existence presque systématique de contenus gratuits, sous une forme ou une autre. De tels contenus demandent à être financés, d'où le recours au mode traditionnel de la presse dans ce cas : la publicité. Une raison supplémentaire, propre à l'internet, tient également à ce que la mise en ligne rend possible une forte diversification des supports de la publicité (bannières, liens sponsorisés, pop-up...), la rendant de ce fait diversement acceptable par les lecteurs.

Plusieurs mouvements convergents incitent les titres de presse à évoluer vers des modèles publicitaires en ligne faisant une large place à la gratuité.

\section{Graphique 5 - Évolution des recettes de la presse}

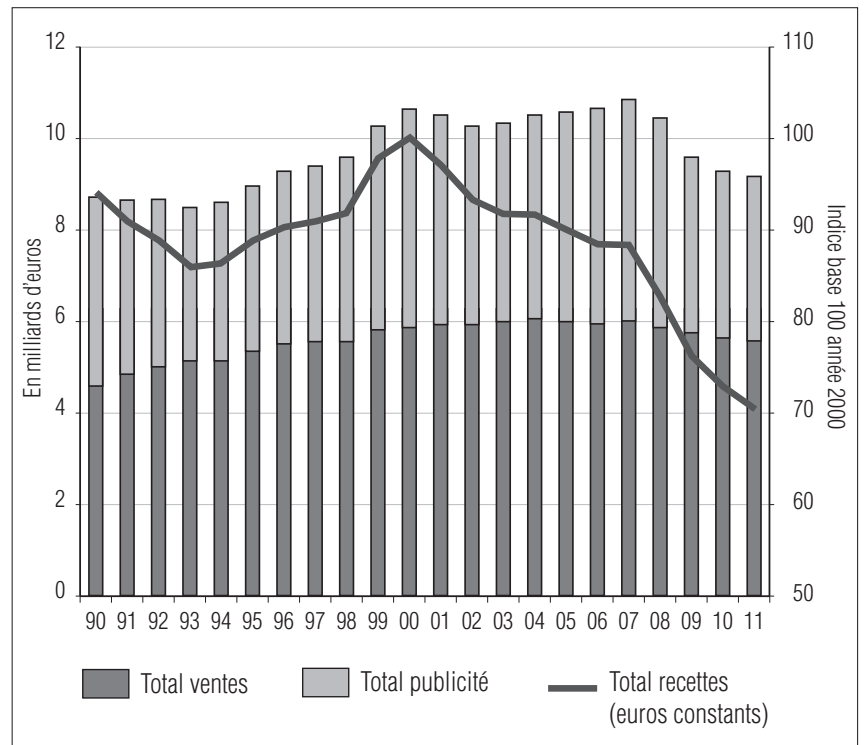

La courbe grise en indice base 100 en 2000 (échelle droite) indique les recettes totales à prix constant sur la période.

Source : http://www.dgmic.culture.gouv.fr

\section{Graphique 6 - Évolution des investissements publicitaires dans la presse papier et numérique, 2003-2011}

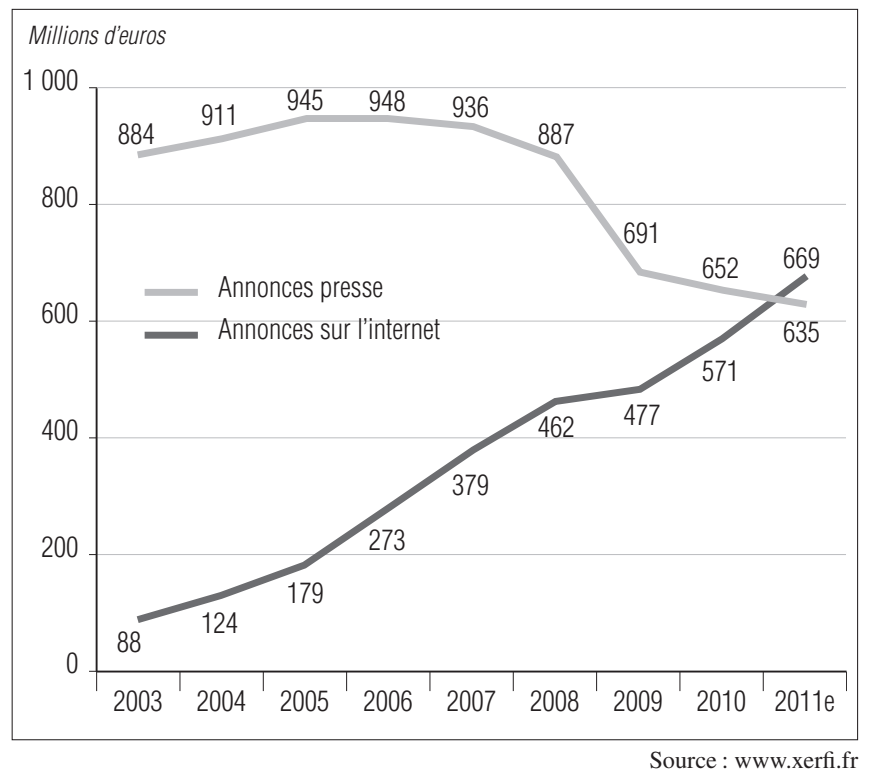

Tout d'abord, les titres doivent répondre à la baisse importante des ventes papier et des revenus publicitaires qui y étaient traditionnellement associés (graphique 5).

Ensuite, un mouvement de bascule des annonceurs conduit à un développement de la publicité et des annonces en ligne au détriment des supports classiques (graphique 6).

Enfin, les titres constatent que les recettes des ventes en ligne sont pour l'instant incapables de compenser l'effet de ciseau associé à la baisse des ventes papier.

\section{Le groupage}

Dans le modèle du groupage ${ }^{16}$, la gratuité tient au fait qu'un produit ou service est fourni gratuitement parce que le consommateur paie, de fait, pour un autre. Ce modèle existe aussi dans la presse papier lorsque la vente du journal est jumelée à différents suppléments : magazines du week-end, livrets ou DVD. Dans la presse numérique, l'offre payante en ligne est souvent couplée à la fourniture des versions papier ou mobile. Dans ce cas, au lieu de vendre les offres distinctes aux différents segments de consommateurs, l'éditeur les vend en un seul ensemble.

\section{Le freemium}

Pour réussir, plusieurs sites de la presse en ligne ont développé et expérimenté une nouvelle modalité de valorisation, que l'on appelle souvent le modèle freemium, de la conjonction des termes anglais premium (contenus de haute qualité) et free (gratuit). Dans ces cas, le site propose gratuitement la mise à disposition d'une partie de son offre seulement. Qui plus est, celle-ci est de qualité réduite (articles anciens, dépêches d'agences ou qualité technique bridée) ou ne correspond qu'à une fourniture restreinte des contenus (extraits de contenus, limitation artificielle des performances ou du débit...). De fait, cette offre gratuite vise à drainer les consommateurs vers des produits ou services payants ${ }^{17}$ à haute valeur ajoutée, en se servant de la gratuité comme d'un produit d'appel. Cet aspect distingue le freemium du groupage. Ce modèle correspond souvent à la démarche des entreprises de presse qui se servent de contenus gratuits comme produit d'appel et essaient de valoriser en ligne les contenus qu'elles finançaient auparavant avec les ventes papier.

\section{Le modèle participatif}

Ce modèle consiste à utiliser la créativité, l'intelligence et le savoir-faire d'un grand nombre d'internautes. Le contenu en ligne est offert gratuitement en échange des contributions des consommateurs qui créent de la valeur (en améliorant le service ou en créant des informations). Pour la presse comme pour d'autres secteurs culturels, le problème qui se pose au site tient moins au choix du mode de valorisation économique des contenus produits, qu'au choix de modèle qui permette une forte implication des lecteurs et une agrégation des contributions de bonne qualité. 


\section{Et les offres d'abondance?}

Une observation intéressante tient au rôle, dans le cas de la presse en ligne, d'un autre modèle économique, souvent discuté et associé dans la littérature à la notion de gratuité : le forfait. Dans ce modèle, le consommateur acquitte un paiement global pour une offre agrégée. Ce faisant, il obtient la possibilité d'un accès illimité qui peut être ressenti comme gratuit ${ }^{18}$ puisque chaque consultation, écoute ou téléchargement ne donne, dès lors, plus lieu à aucune transaction. Même si ce modèle a été, ces dernières années, largement popularisé dans d'autres secteurs, il reste rare dans le cas de la presse numérique. Lorsqu'il s'agit de l'offre en ligne, tout ou partie des contenus sont publiés sur le site pour signaler la qualité et inciter les lecteurs à payer les services supplémentaires ou le contenu de meilleure qualité. En d'autres termes, dans le cas de la presse en ligne, ce modèle se rapproche de ceux du groupage ou du freemium.

Les quatre modèles numériques de gratuité (publicité, groupage, freemium, modèle participatif) s'inscrivent de façon inédite dans les trois classes des modèles d'affaires (graphique 7).

Ainsi par exemple, les acteurs de la presse française de la troisième classe, les leaders explorateurs, favorisent les modèles de freemium (commercialisation des contenus et des services à haute valeur ajoutée), de groupage (abonnement web+, abonnement web/papier, etc.) et de publicité.

La classe des pure players a pour caractéristique principale l'articulation du modèle participatif (59\% des titres), et du modèle de publicité ( $71 \%$ des titres, y compris $18 \%$ qui emploient le modèle entièrement fondé sur la publicité sans recours aux ventes directes des contenus aux consommateurs finaux).

Par ailleurs, nos résultats montrent que le modèle participatif est rarement combiné avec le modèle freemium et le modèle groupage. Cependant, le cas de Médiapart constitue un contre-exemple et prouve que ces approches ne sont pas incompatibles.

Accessoirement, chez les pure players (par exemple, Streetpress ou Rue89), on constate la commercialisation des biens et services indépendants de l'offre principale : vente de bandes dessinées, dictionnaires, livres, produits électroniques, formation et conseil, etc.

Enfin, le recours aux modèles de gratuité en ligne dans la première classe des numériques a minima est relativement faible: le modèle le plus fréquent, celui de la publicité, est employé seulement par $37 \%$ des éditeurs de presse. Par ailleurs, $43 \%$ des titres de presse de cette classe ne génèrent pas de revenus en ligne et financent leur site par les ventes papier.

Ainsi, il existe une multiplicité de modèles de gratuité dans la presse et le modèle publicitaire n'est pas le seul mis en œuvre par les acteurs. Les choix stratégiques déterminant les différents modèles de gratuité sont liés, au moins en partie, au positionnement des titres de presse (graphique 8). Cela s'explique par le fait que les acteurs dans la presse sont confron- tés, selon leur positionnement, à des difficultés de nature variée. Par exemple, les magazines (bi)hebdomadaires ou (bi)mensuels, grâce au ciblage des publics, sont parvenus à préserver leur diffusion mais leur offre commerciale souffre du

\section{Graphique 7 - Inscription des modèles de gratuité dans les trois classes de modèles d'affaires}

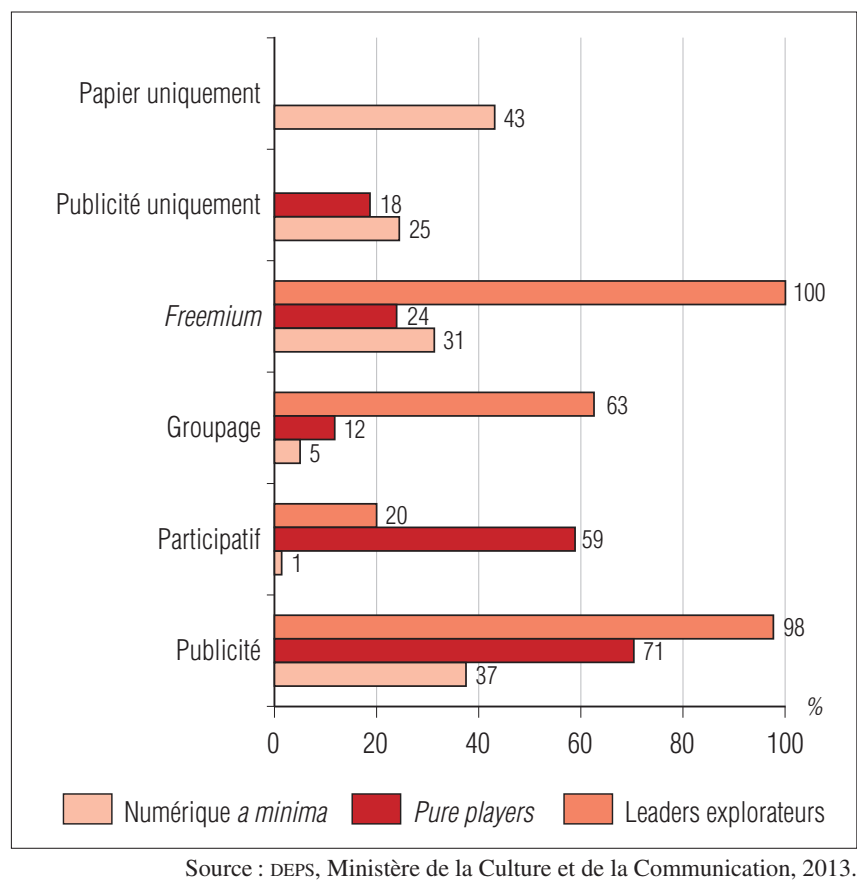

\section{Graphique 8-Choix de modèle de gratuité en fonction de la fréquence de publication}

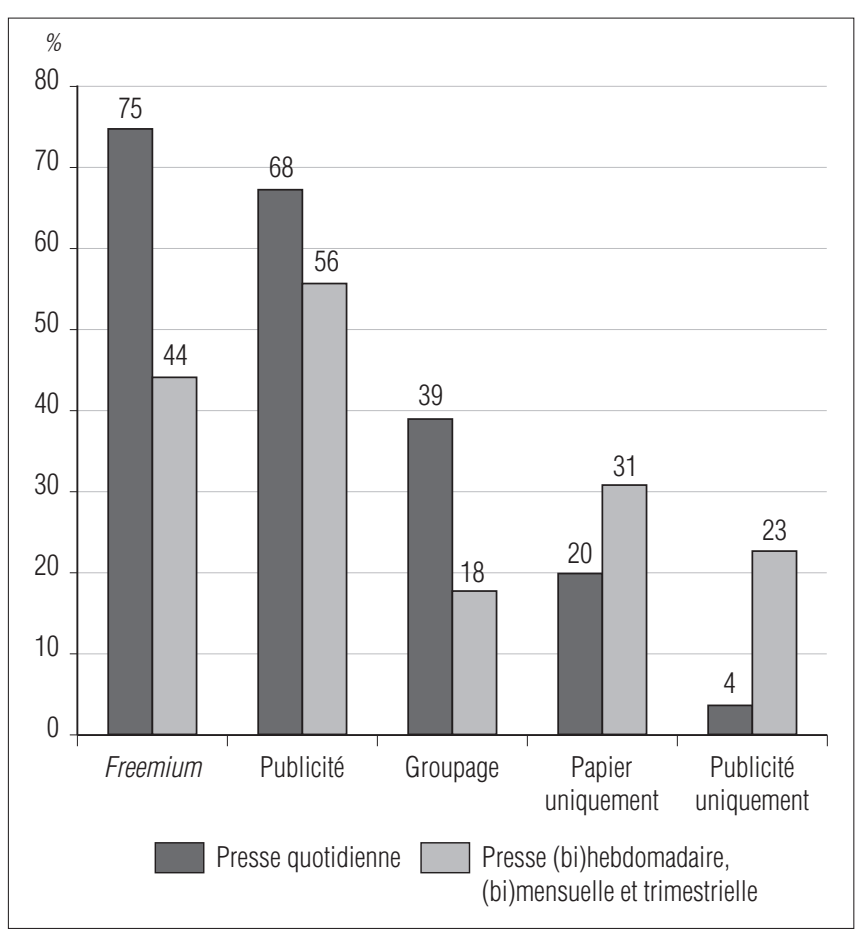

Source : DEPS, Ministère de la Culture et de la Communication, 2013. 
manque de réactivité qu'impose leur périodicité (par rapport aux autres contenus en ligne), ce qui ralentit leur adaptation aux nouveaux contextes. La presse quotidienne, compte tenu de la chute des revenus publicitaires, cherche à valoriser en ligne les contenus qu'elle finançait initialement avec les ventes papier et pour lesquels elle tente de trouver des ressources complémentaires en ligne. Finalement, les nouveaux médias en ligne (pure players) indépendants (ou presque) des groupes de presse souffrent souvent de revenus insuffisants, en n'étant pas suffisamment suivis par les annonceurs. Ils cherchent donc à diversifier leurs activités et à occuper de nouvelles niches de la presse participative et citoyenne.

\section{Conclusion}

Les nouvelles configurations appellent, de la part des acteurs des industries culturelles, des stratégies inédites et de nouvelles manières de construire leurs positions compétitives. La concurrence entre diffuseurs, voire entre structures intégrées, tend ainsi parfois moins à s'opérer sur le projet éditorial ou sur le choix des œuvres elles-mêmes que sur les modèles d'affaires et les moyens de les mettre à disposition du public. Dans de tels cas, les stratégies à l'œuvre visent la captation de l'audience, la croissance et la part de marché plus que la rentabilité.

Combien de sites ne défendent-ils et ne justifient-ils pas ainsi la pertinence de leur modèle économique par le nombre de visiteurs, de campagnes publicitaires, de pages vues... sans mentionner le chiffre d'affaires ou la performance économique engendrée par ces résultats d'audience. Cette situation aboutit en définitive à une forte opacité sur les prix et les conditions de rentabilité des activités, donnant tout leur poids aujourd'hui aux dynamiques de la gratuité.

Pourtant, si gratuité il y a, elle s'opère toujours parallèlement à une génération de revenus. Le cas de la presse montre que, selon les cas, le recours à la gratuité correspond néanmoins à une valorisation très différente de l'activité et des ressources de l'organisation productive. On peut fournir des services gratuitement pour générer du trafic ou bien pour alimenter des ressources annexes ou encore pour élargir et consolider un marché, voire comme contrepartie à des subventions. Ces différentes formes de gratuité s'accompagnent, parallèlement, de différents modes de rémunération : freemium, groupage, publicité, ventes d'information à des tiers... C'est d'ailleurs l'une des limites de l'étude que de n'avoir pu disposer, pour chaque titre, de la structure des revenus effectivement générés, ce qui n'a pas permis de préciser l'importance respective des modèles et les modalités de leur articulation.

Derrière le terme de gratuité, la net économie révèle des dynamiques très différentes. Une telle variété n'est en aucun cas garante de la soutenabilité et de la viabilité de l'e-gratuité. $\mathrm{Au}$ contraire, elle est sans doute le signe de ses grandes incertitudes. E-gratuité et e-rentabilité cohabitent désormais. Sauf à imaginer, dans un effet de domino suicidaire, une généralisation progressive de la gratuité à tous les acteurs de la chaîne de valeur, la question se pose aujourd'hui de savoir quand le marché se stabilisera et épousera, en le faisant évoluer, les modèles économiques industriels classiques.
L'analyse des différents modèles de la presse en ligne réinterroge les formes d'intervention et de soutien publics. La variété des modèles à l'œuvre appelle, en particulier, à considérer que la structuration des titres de presse ne peut plus être simplement envisagée à partir de leurs caractéristiques traditionnelles, décalquées des modalités de diffusion papier (périodicité, aire de diffusion ou thématique des informations notamment). Les classes identifiées montrent au contraire que les nouvelles stratégies qui se dessinent sur l'internet traversent justement ces catégories traditionnelles. Le même constat s'applique aussi au traitement de la gratuité. La variété des formes observables et des stratégies à l'œuvre parmi les titres devrait inciter à moduler de manière spécifique l'application d'accords éventuels de branche face aux acteurs puissants de l'internet. Faut-il, par exemple, s'en servir pour protéger les modèles traditionnels de la presse, comme le font les numériques $a$ minima, ou au contraire pour accompagner l'ensemble des titres vers de nouvelles formes d'organisation et de valorisation, à l'image de ce que recherchent les leaders explorateurs? Quelle place enfin, dans ce nouveau paysage, pour les nouveaux acteurs de la presse en ligne : ont-ils vocation à être éligibles - et sur quelles bases - aux politiques publiques de soutien à la presse ? 


\section{Numérique a minima : 81 titres}

6 Mois

Bambi

Bilto

Bordeau 7

Bretons

Centre presse Aveyron semaine

Charlie hebdo

Direct Lille plus

Direct Lyon plus

Direct Marseille plus

Direct Matin plus

Direct Montpellier plus

Direct Nantes + Direct Strasbourg

+ Direct Toulouse

Direct Soir

Elle à table

Eure infos

Gala

Informations ouvrières

L'Actu

La Dordogne libre

La Gazette de Côte-d'or

La Gazette de l'Ognon + La Gazette du

Lion + La Gazette du Ballon + Le Petit Messager

La Presse d'Armor

La Presse de la Manche

La République des Pyrénées
La Vie corrézienne

Le Canard enchaîné

Le Courrier cauchois

Le Courrier de Fourmies-L'Observateur de l'Avesnois

Le Courrier de la Mayenne

Le Courrier du pays de Retz

Le Courrier S'Blattel

Le Courrier-La Gazette

Le Criquet magazine

Le Démocrate indépendant de Bergerac

L'Écho du Pas-de-Calais

L'Écho-Le Régional d'Enghien

L'Éclaireur de Gamaches

Le Favori de la presse

Le Monde libertaire

Le Petit Quotidien

Le Tarn libre

Les Infos-Pays de Ploermel

Les Inrockuptibles

Les Nouvelles d'Anjou

L'Indépendant de l'Yonne

L'Observateur de l'Arrageois

L'Observateur du Cambresis

L'Observateur du Douaisis

L'Observateur du Valenciennois

Lutte ouvrière hebdo/i commme info

Magazine de l'ape
Mes premiers J'aime lire

Métro

Métro Nice-Cannes

Mickey parade géant

Minute

Mon quotidien

New Stars Mag

Notre temps

Paris courses

$\mathrm{PC}$ achat

Pleine vie

Présent

Revue XXI

Rivarol

La Sambre-La Frontière

Télé 2 semaines

Télé 7 jours (première)

Télé loisirs

Télé star

Télé z

Thierache

Tiercé magazine

Top vélo

Tout est à nous

TV grandes chaînes

TV magazine

Version fémina

Week-end

\section{Pure players : 17 titres}

20 Minutes

Agora Vox

Arrêt sur images

Atlantico

France net infos

France soir
Jolpress

Le Post

Les Nouvelles News

Médiapart

Newsring

Owni
Quoi.info

Rue89

Slate

Streetpress

The Pariser

\section{Leaders explorateurs : 51 titres}

Aujourd'hui en France

Berry républicain

Corse matin

Courrier international

Dossier familial

Femme actuelle

Investir

Journal de la Haute-Marne

La Croix

La Dépêche du Midi

La Manche libre

La Montagne

La Nouvelle République des Pyrénées

La Provence

La Recherche

La République du Centre

La Tribune
La Tribune républicaine de Bellegarde

La Voix du Nord

L'Écho républicain de Chartres

Le Figaro

Le Journal du Centre

Le Journal du dimanche

Le Monde

Le Nouvel Observateur

Le Pays gessien

Le Point

Le Populaire du Centre

L'Équipe

Les Dernières Nouvelles d'Alsace

Les Échos

L'Essor savoyard 73

L'Essor savoyard 74

L'Est républicain
L'Express

L'Humanité

Libération

L'Indépendant

Marianne

Messager Thonon

Midi Libre semaine

Ouest France

Paris Match

Paris Normandie

Paris turf

Politis

Sud Ouest

Télérama

Var matin

Vosges matin

Yonne républicaine 


\section{Éléments de méthodologie}

\section{Graphique 9 - Répartition de l'ensemble des titres de la presse française en 2012}

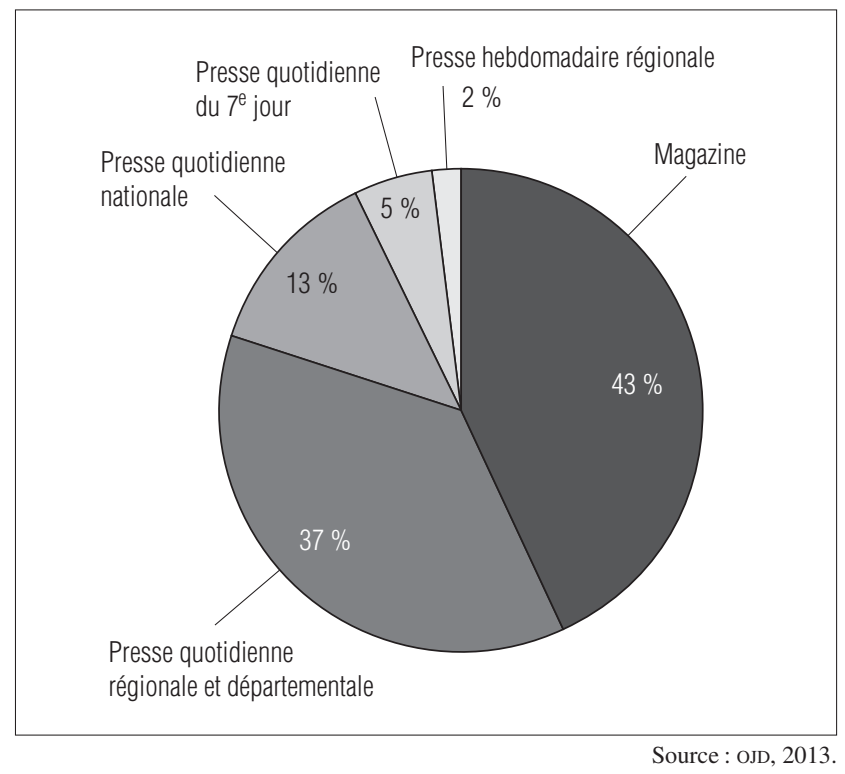

Dans un premier temps, les sites web de la presse française ont été recensés puis caractérisés grâce à une spécification de leur classement à travers différentes dimensions: (1) catégorie institutionnelle, localisation, thématique, fréquence de publication; (2) contenus et services offerts en ligne; (3) dispositifs d'interaction avec les lecteurs ; (4) dispositifs de stockage ; (5) réseau de production du contenu ; (6) dispositifs de distribution ; et (7) stratégies de tarification.

Ce premier travail a permis la constitution d'une base de données inédite et particulièrement riche sur les sites web de 149 acteurs de la presse en ligne française. Le choix des acteurs s'est effectué sur la base des données de l'Association pour le contrôle et la diffusion des médias (http://www.ojd.com/). Ces acteurs ont été choisis en s'appuyant sur les typologies mobilisées usuellement, afin de garantir la représentativité en termes de catégories institutionnelle, thématique, fréquence de publication, localisation et taille des titres français.

Dans un deuxième temps, une démarche statistique exploratoire multidimensionnelle a été mobilisée pour une caractérisation et une typologie des modèles d'affaires. La typologie des modèles d'affaires de la presse numérique a été construite à partir d'une analyse de correspondances multiples, suivie d'une classification hiérarchique ascendante. Ces deux méthodes sont des méthodes de l'analyse multivariée. La première méthode représente une extension de l'analyse factorielle des correspondances (AFC) pour l'étude simultanée de plusieurs variables. La visualisation du plan factoriel présente l'information contenue sur les deux premiers axes factoriels (graphique 1). Le nombre total des axes retenus pour l'analyse (5 axes) est choisi en fonction de l'inertie totale du nuage de points - variables et individus - et des objectifs méthodologiques (identifier les classes pertinentes des modèles d'affaires).

La méthode de classification hiérarchique ascendante a pour objectif d'obtenir des classes d'individus les plus cohérentes possibles en formant les groupes les plus homogènes. Grâce à cette méthode, les variables discriminantes entre les classes, les caractéristiques les plus importantes par classe et les individus les plus représentatifs de chaque classe de modèle d'affaires peuvent être identifiés.

\section{RÉSUMÉ}

Sous l'impact des nouvelles technologies d'information et de communication (TIC), on observe ces dernières années dans l'industrie de presse une multiplication des configurations des formes organisationnelles et des modèles d'affaires. La multiplication de ces modèles ou des manières de mettre les contenus à disposition ne constitue pas un simple effet conjoncturel. Il traduit des stratégies systématiques d'innovation et d'exploration de modèles d'affaires alternatifs, qui représentent aujourd'hui la source d'avantage concurrentiel privilégiée. L'analyse aborde la variété des nouveaux modèles d'affaires par un effort de catégorisation et de description permettant d'identifier et de comprendre les trajectoires d'évolution de l'économie de la presse. L'étude économétrique d'une base de données inédite de 149 titres de presse française a permis d'identifier trois classes distinctes de modèles d'affaires de la presse en ligne : numérique a minima, pure players et leaders explorateurs.

\section{ABSTRACT}

In recent times the press industry has been reshaped by the influence of Information and Communication Technologies (ICT). Constant experimentation and innovation in the business model (BM) has become one of the key sources of actors' competitive advantage. As a result, dominant and stable traditional BMs have given rise to multiple disruptive BMs. The goal of this study is to provide typology and description of the new online BMs and to understand the current economic transformations in the press industry. The analysis relies on the empirical study of 149 French press websites accounting for the various configurations of digital output and technology in production processes. Three classes of online BMs were identified: the "minimally digital", "pure players" and "exploring leaders". We substantiate the features of the three classes of online BMs and put forward new relevant approaches to the "free" content funding.

Pour recevoir régulièrement les publications du DEPS et pour toute demande d'information : contact.deps@culture.gouv.fr 